\title{
Paracoccidioidomycosis simulating cancer diagnosed by endoscopic ultrasound-guided fine needle aspiration
}

A 57-year-old woman presented with abdominal pain, jaundice, and a $12-\mathrm{kg}$ weight loss over the preceding 6 months. The abdominal computed tomography (CT) scan demonstrated a mass located in the head of pancreas $(6.4 \times 5.8 \mathrm{~cm})$. Endoscopic retrograde cholangiopancreatography (ERCP) was performed for biliary drainage with the insertion of a single 10-Fr plastic stent in the common bile duct (CBD), which led to an improvement in her jaundice.

Furthermore, multiple enlarged lymph nodes were seen in the hepatic hilum on magnetic resonance imaging (MRI; - Fig. 1), but there was no evidence of the previously visualized pancreatic mass. An endoscopic ultrasound (EUS) showed multiple enlarged lymph nodes in the hepatic hilum ( Fig.2), splenic hilum, and celiac trunk, adjacent to the pancreas and $\mathrm{CBD}$, but no pancreatic lesion. During EUS-guided fine needle aspiration (EUS-FNA) of the hepatic hilum lymph nodes using a 22-gauge needle (Echo Tip; Cook Medical, Winston-Salem, North Carolina, USA; Fig. 3), a purulent fluid was released and collected for further analysis. The histopathological analysis revealed Paracoccidioides brasiliensis spores ( Fig.4).

Paracoccidioidomycosis is a systemic mycosis caused by the dimorphic fungus $P$. brasiliensis. The disease is restricted to Latin America. It is the main systemic mycosis seen in Brazil, with higher incidences found in the central and southern regions. The disease is acquired by inhaling fungal propagules [1].

Paracoccidioidomycosis has several clinical presentations depending on the affected organ. Lymph node involvement is usually secondary to a skin and/or visceral primary focus and affects predominantly the cervical and supraclavicular lymph node chains. Other findings are irregularity of the pancreatic head that can simulate cancer, biliary duct dilatation with cholestasis, abscesses, and splenic calcifications, among others [2]. Abdominal lymph node involvement simulating cancer is rare [3]. The use of EUS-FNA to make this diagnosis is even rarer.

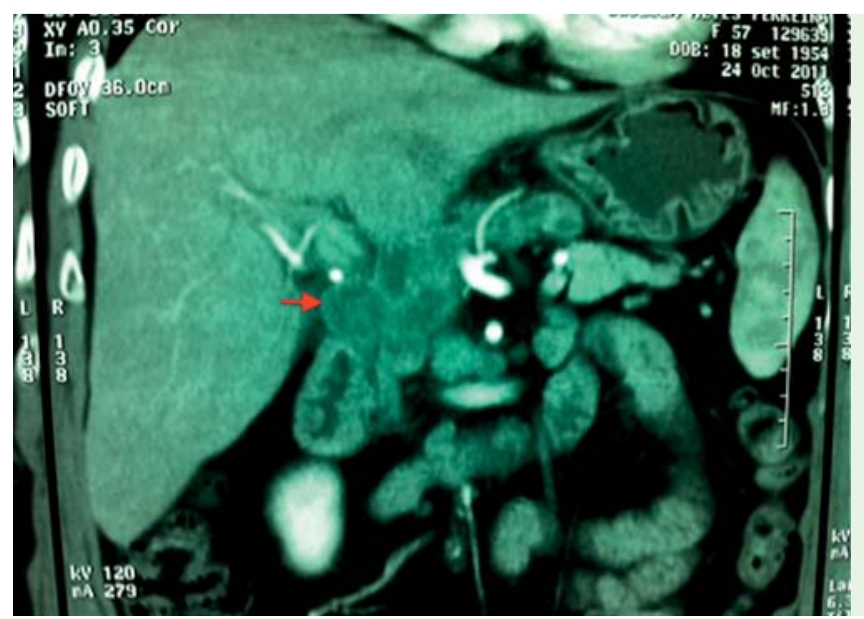

Fig. 1 Abdominal magnetic resonance imaging (MRI) scan in a 57-year-old woman with jaundice and weight loss showing multiple enlarged lymph nodes at the hepatic hilum (arrow).
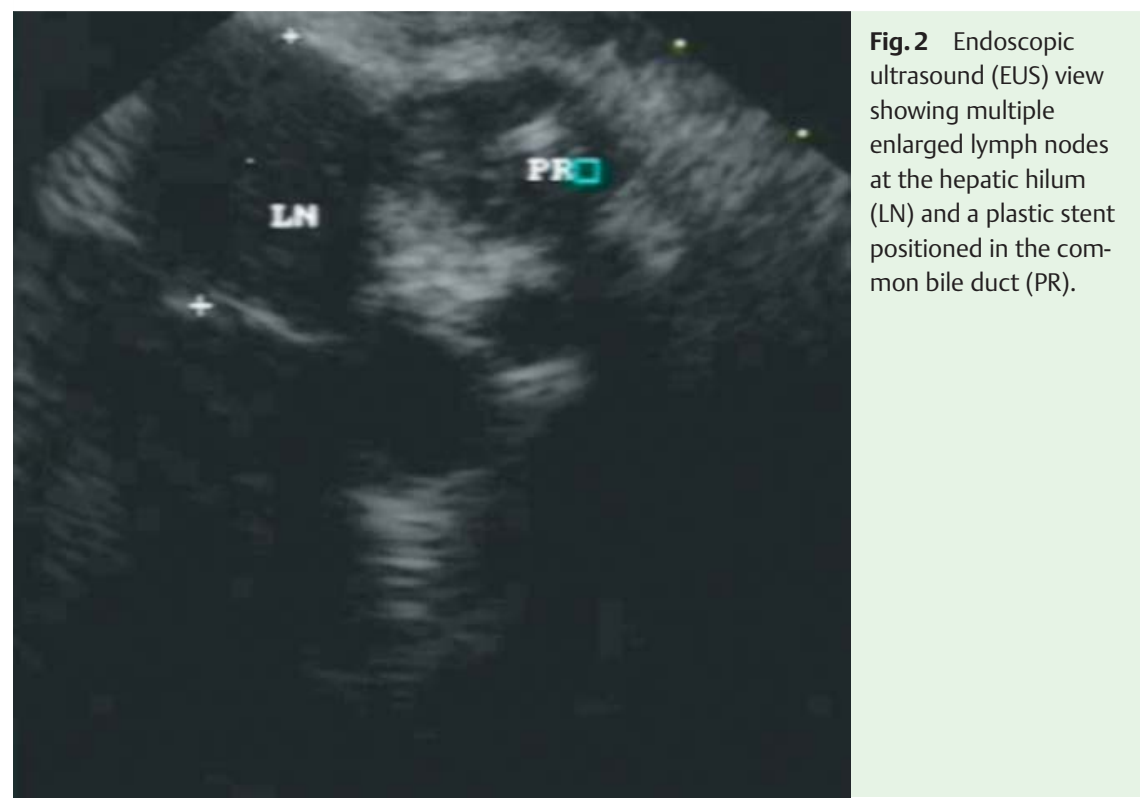

Our group previously published the first case of adrenal paracoccidioidomycosis diagnosed by EUS-FNA [4]. To our knowledge, this is the first reported case of paracoccidioidomycosis with abdominal lymphadenopathy simulating cancer having been diagnosed by EUS-FNA.

Endoscopy_UCTN_Code_CCL_1AF_2AG_3AD

G. R. Silva Neto ${ }^{1}$, R. L. Ganc ${ }^{1,2}$, R. Colaiacovo ${ }^{1,2}$, F. K. N. Bueno", F. C. Bernardi ${ }^{3}$, L. G. B. Rossini ${ }^{1}$

${ }^{1}$ Department of Endoscopy, Santa Casa University Hospital, São Paulo, Brazil

${ }^{2}$ Department of Endoscopy, Albert Einstein Jewish Hospital, São Paulo, Brazil ${ }^{3}$ Department of Pathology, Santa Casa University Hospital, São Paulo, Brazil

\section{Competing interests: None}



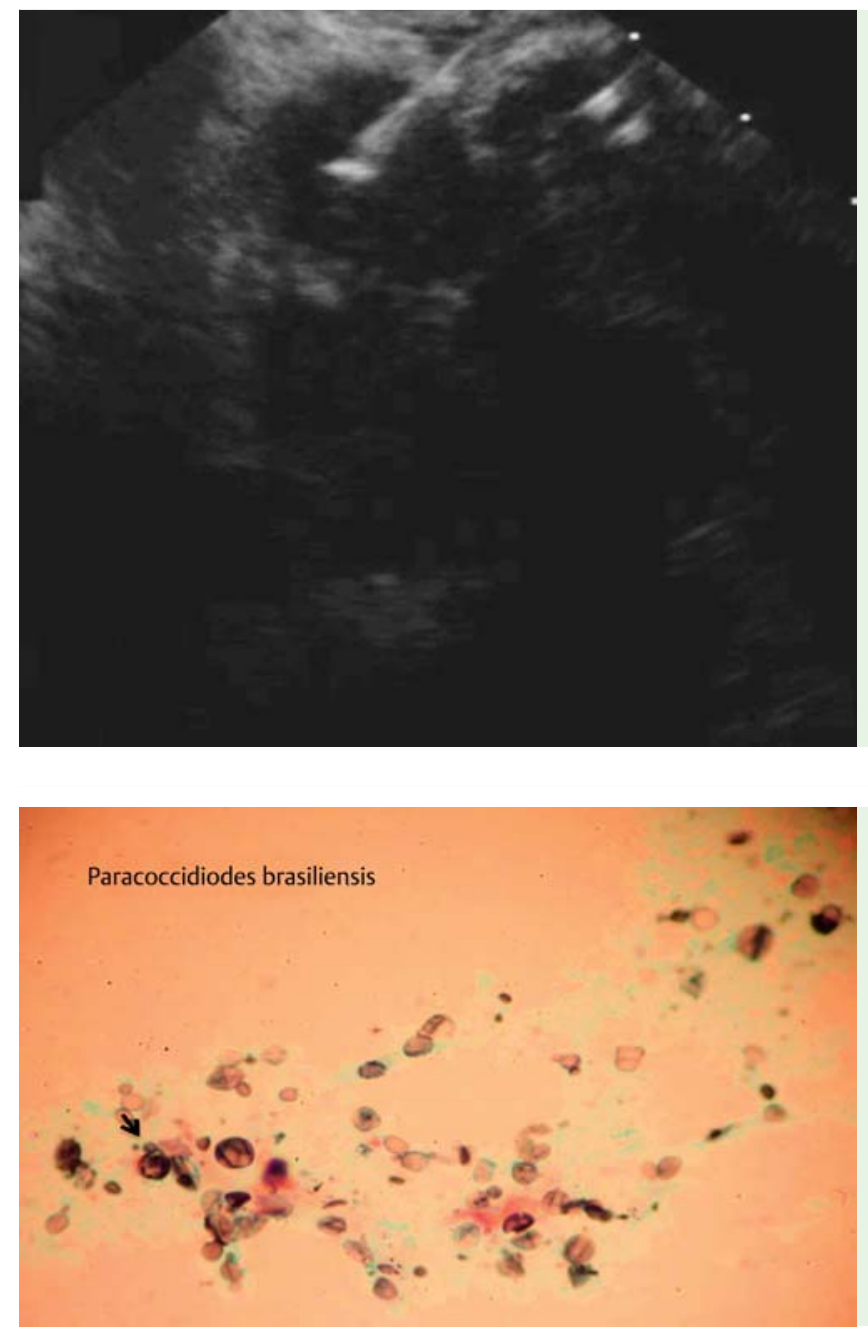

Fig.3 Image during endoscopic ultrasoundguided fine needle aspiration (EUS-FNA) of a lymph node at the hepatic hilum.

\section{References}

1 Wanke B, Aidê MA. Paracoccidioidomycosis. J Bras Pneumol 2009; 35: 1245 - 1249

2 Martinez R, Bellucci $A D$, Fiorillo AM. Computerized tomography in the evaluation of abdominal involvement in paracoccidioidomycosis. Mycoses 1988; 31: 187-197

3 Pereira PMR, Akel PBM, Lima LL et al. Multifocal paracoccidioidomycosis: a diagnostic challenge due to late cutaneous manifestation. An Bras Dermatol 2011; 86: 149-152

4 Colaiacovo R, Ganc RL, Leone AC et al. Diagnosis of left adrenal paracoccidioidomycosis by endoscopic ultrasound fine needle aspiration. Endoscopy 2011; 43: E236-E237

\section{Bibliography}

DOI http://dx.doi.org/

10.1055/s-0032-1325897

Endoscopy 2012; 44: E439-E440

(c) Georg Thieme Verlag KG

Stuttgart · New York

ISSN 0013-726X

Fig. 4 Histopathological staining of the exudate collected during endoscopic ultrasound-guided fine needle aspiration (EUS-FNA) showing the multiple spores (arrows; Grocott's silver stain; magnification $\times 100$ ).

\section{Corresponding author}

\section{G. R. da Silva Neto, MD}

Santa Casa of São Paulo Medicine School Endoscopy

Rua Cesário Motta Junior 112

Santa Cecília São Paulo

São Paulo 01221-020

Brazil

Fax: +55-11-21767236

gabriel-med@hotmail.com 